\title{
The name of deliciousness and the gastrophysics behind it
}

\author{
Ole G Mouritsen ${ }^{1 *}$, Lars Duelund ${ }^{1}$, Luis A Bagatolli ${ }^{2}$ and Himanshu Khandelia ${ }^{1}$
}

\begin{abstract}
The term 'gastrophysics' has been proposed to describe an emerging scientific discipline that employs an arsenal of the most powerful theoretical, simulational, and experimental techniques from the physical sciences to study the empirical world of cooking and gastronomy. In the same way that biology has inspired the field of biophysics, gastronomy is the source of inspiration for gastrophysics. In particular, gastrophysics aims at exploiting recent advances in the physical sciences to forward the scientific study of food, the raw materials used, the effects of processing food, and quantitative aspects of the physical basis for food quality, flavor, appreciation, and absorption in the human body. In this study, we focused on questions pertaining to the texture and flavor of a particular type of raw material, namely, the red seaweed, dulse (Palmaria palmata), and demonstrate how a combination of physical chemistry, biophotonics, and atomic-scale molecular simulation might shed some light on these questions, particularly in relation to the physical mechanism of the umami sensation.
\end{abstract}

Keywords: Gastrophysics, Umami, Seaweeds, Receptor, Synergy

\section{Gastrophysics research program}

We and our colleagues at the Center for Biomembrane Physics have a wide range of interests, competencies, and facilities in the broad field of biophysics, and in the physics and physical chemistry of biological materials. In particular, we are interested in lipids, proteins, and membranes, as well as of the different phenomena related to membranes, including membrane function, the action of drugs, and the effects of various processes on membranes. Moreover, we have available a large arsenal of theoretical, experimental, and simulational methods and instrumentation. Taken together with a keen interest in food and the enjoyment of food, a natural step was to apply all these capacities to study problems related to gastronomy. After all, the empirical world of cooking mostly uses materials of biological origin, the methods used in cooking involve some kind of chemical and physical processing [1-5], and the sensations of food, such as mouth-feel and flavor, are based on mechanisms obeying principles from physics and chemistry.

\footnotetext{
* Correspondence: ogm@memphys.sdu.dk

${ }^{1}$ MEMPHYS, Center for Biomembrane Physics, Department of Physics,

Chemistry, and Pharmacy, University of Southern Denmark, Campusvej 55,

Odense M DK-5230, Denmark

Full list of author information is available at the end of the article
}

An initial interest in food from the sea, stimulated by the first author's passion for Japanese cuisine, particularly sushi [6], gradually developed into questions regarding the use of marine macroalgae (seaweeds) [7], and from there to the flavor of certain seaweeds, in particular the taste of deliciousness (umami) [8,9]. The work was greatly stimulated by collaboration with a range of inventive chefs.

We provide a brief preliminary report on the likely results to which such an approach can lead, and hence, by example, give an opinion on what gastrophysics could be.

\section{Flavor of seaweeds}

The history of flavor owes so much to a discovery made by Kikunae Ikeda, who in 1908 [10] studied the chemical composition of the large brown seaweed, konbu (Saccharina japonica). Konbu is used together with a highly processed fish product, katsuobushi, to produce dashi, the soup broth around which the entire Japanese cuisine revolves. Ikeda found that konbu contains very large amounts of free monosodium L-glutamate (MSG). He suggested that this compound is the primordial source, or the essence of deliciousness (umami), and he proposed it as a fifth basic taste 
quality, in addition to the existing four qualities of salt, sour, sweet, and bitter. However, it was only in the early part of this century, after the discovery of specific umami receptors located in the taste-cell membranes, that umami was fully recognized as a basic physiological taste sense [11-15].

Seaweeds are a common staple of Asian cuisine, not only in Japanese cooking, but they are rarely used nowadays in western cuisine [16]. We set out to explore whether Nordic seaweeds are equally flavorful, and discovered that the red seaweed species, dulse (Palmaria palmata), which has been used for centuries in traditional cuisine in Ireland, Brittany, and Iceland, also has large amounts of MSG [17]. To assess its flavor, we developed various extraction methods, inspired by processes in the kitchen, and used physicochemical techniques to assess the amino-acid profiles of the extracts under different conditions of temperature and composition (an example of the results of such an analysis is shown in Figure 1). We found that dulse has very large amounts of free MSG as well as other flavorful amino acids, such as aspartic acid (umami sensation); alanine, proline, glycine, and serine (all sweet); and measurable amounts of isoleucine, leucine, and valine (all bitter). Indeed, the umami capacity of dulse is comparable to that of good quality konbu $[17,18]$. Together with several chefs, we explored the possibilities of dulse-derived dashi in various dishes, including ice cream, bread dough, and fresh cheeses [17].

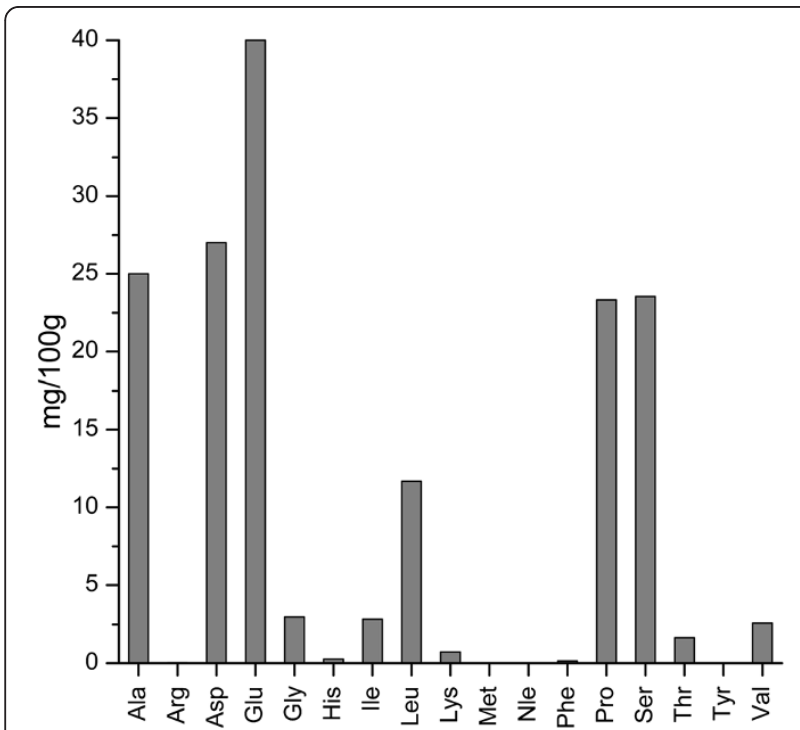

Figure 1 Amino-acid profile for dashi made from the red seaweed dulse. The dashi was produced by using the sous-vide technique at $60^{\circ} \mathrm{C}$, resulting in an extract of $10 \mathrm{~g}$ dry dulse in $500 \mathrm{ml}$ water, which was analyzed by mass spectrometry. Data from Mouritsen et al. [17].

\section{Texture of seaweeds}

Seaweeds also play another important, but little-known role in cooking. Their contents of certain complex polysaccharides particularly alginate, agar, and carrageenan, readily form stable hydrogels and are highly valued as gelling agents, providing texture to foodstuffs and enhancing the mouth-feel of fluid materials. These polysaccharides are used by the seaweeds to bind together their cells that in most species are little specialized. The polysaccharides can be extracted from the seaweeds by chemical methods, thus alginate, agar, and carrageenan introduced into foodstuff have to be declared as food additives.

However, there is no reason why raw seaweeds that have been appropriately processed (for example, as a fine particulate material), could not be used as a whole ingredient in food. In this case, not only can the gelation properties of the seaweeds be exploited, but also their flavor characteristics. The gastrophysical questions that arise in this context pertain to seaweed structure and texture under different preparation methods. In this project, we used advanced bioimaging techniques to study the cellular structure of seaweed under different conditions of hydration and processing, such as aging and cooking. Figure 2 shows an example of a fully hydrated frond of dulse, in which the large intercellular space containing the polysaccharides can be clearly seen. Using various intrinsic and extrinsic fluorescence probes, we

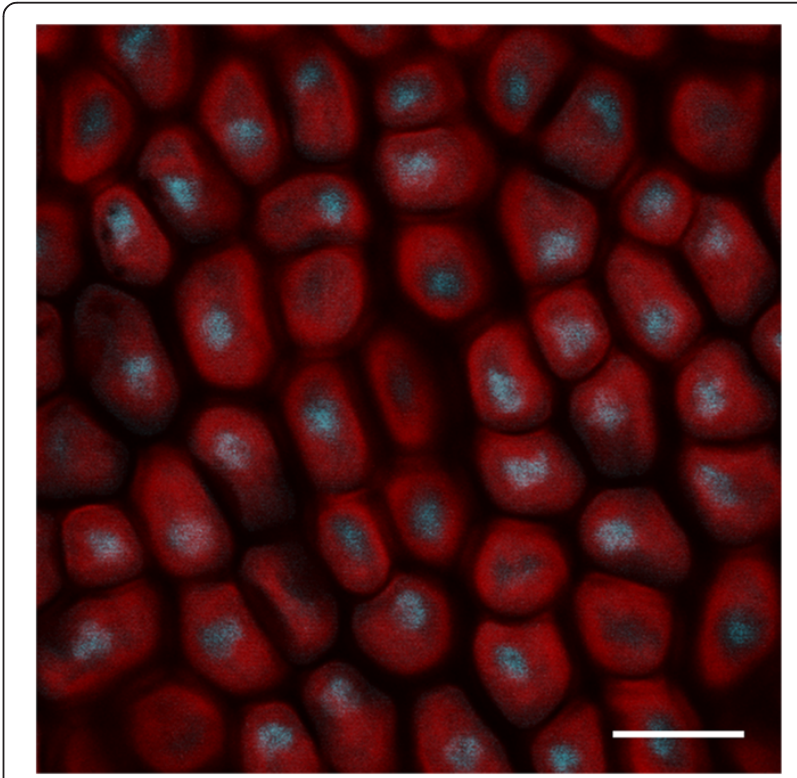

Figure 2 Bioimaging of seaweeds. Two-photon laser microscopy image of a rehydrated frond of the red seaweed dulse (Palmaria palmata), showing a single cross-section imaged by both intrinsic fluorescence and a label (4',6-diamidino-2-phenylindole; DAPI) that stains the DNA of the cell nuclei. The excitation wavelength was $780 \mathrm{~nm}$. Scale bar $10 \mu \mathrm{m}$. 
could image different parts of the cells and map out the structural and spectral properties of the intracellular water.

\section{The molecular mechanism of the umami receptor} Our work on the flavor of seaweeds led to questions regarding the molecular mechanism of the umami sensation, particularly the special synergy that pertains to the simultaneous presence of glutamate and certain $5^{\prime}$-ribonucleotides. The unique aspect of the umami taste is that it can be enhanced many times by the presence of free nucleotides such as inosine $5^{\prime}$-monophosphate (IMP), guanosine $5^{\prime}$-monophosphate (GMP), and adenosine $5^{\prime}$-monophosphate (AMP). Free glutamate is found in large amounts in cured hams, anchovy paste, ripe tomatoes, walnuts, hard and mature cheeses, and soy and fish sauces, as well as certain brown seaweeds. Free nucleotides are abundant in meat broths, chicken, fish and shellfish, mollusks, and dried fungi. In the classical Japanese dashi, the seaweeds provide free glutamate and the katsuobushi free IMP. In shojin ryori, the vegan Japanese temple cuisine, the fish is replaced by shiitake mushrooms, which contain large amounts of free GMP [18-20].

A deeper understanding of this remarkable synergy in umami sensation requires insight into the structure and functioning of the umami receptors that reside in the taste-cell membranes [21]. Although three different kinds of umami receptors have been discovered since 2000, one particular heterodimer G-protein coupled receptor, T1R1/T1R3, is most likely to hold the key to the synergy in umami.

By using large-scale molecular dynamics simulation techniques, we recently studied the atomic-scale dynamics of the Venus-fly-trap domain (VFTD) of the T1R1/ T1R3 umami receptor monomer, both in the presence and absence of glutamate and GMP, in order to unravel the molecular mechanism behind the synergistic effect of these two ligands on the dynamics of the receptor [22]. We found that the dynamics of the VFTD along the hinge-bending motion that activates signaling was dampened significantly after binding of glutamate. The dynamics were further slowed by binding of GMP at an allosteric site, thus suggesting a molecular mechanism of cooperativity between GMP and glutamate (this effect is illustrated schematically in Figure 3).

\section{Conclusion and future outlook}

We consider that gastrophysics has as its goal to demonstrate that fundamental principles of physics, in particular soft-matter physics, biophysical chemistry, and molecular biophysics, can be brought together to work within the sciences dealing with food. In this paper, we give examples of how questions stimulated by an interest in gastronomy can lead to new insights that both have scientific merit in themselves and can have an influence on gastronomy.

For this study, we focused attention on a littleexplored material in the context of gastronomy, namely seaweed [16], and found that this material has very interesting flavor and texture properties. Moreover, using a detailed and well-defined molecular model, our molecular simulations have established, quantitative support for the proposed and putative mechanism [23] behind the ubiquitous synergy effect in the umami sensation. The power of this synergy for cooking delicious food has been known and used by cooks for centuries, and in 1960, it was shown that inosinate from fermented fish or guanylate from dried shiitake together with glutamate from dried seaweeds provided enhanced umami flavor in soup broths [24]. In this study, we have revealed that

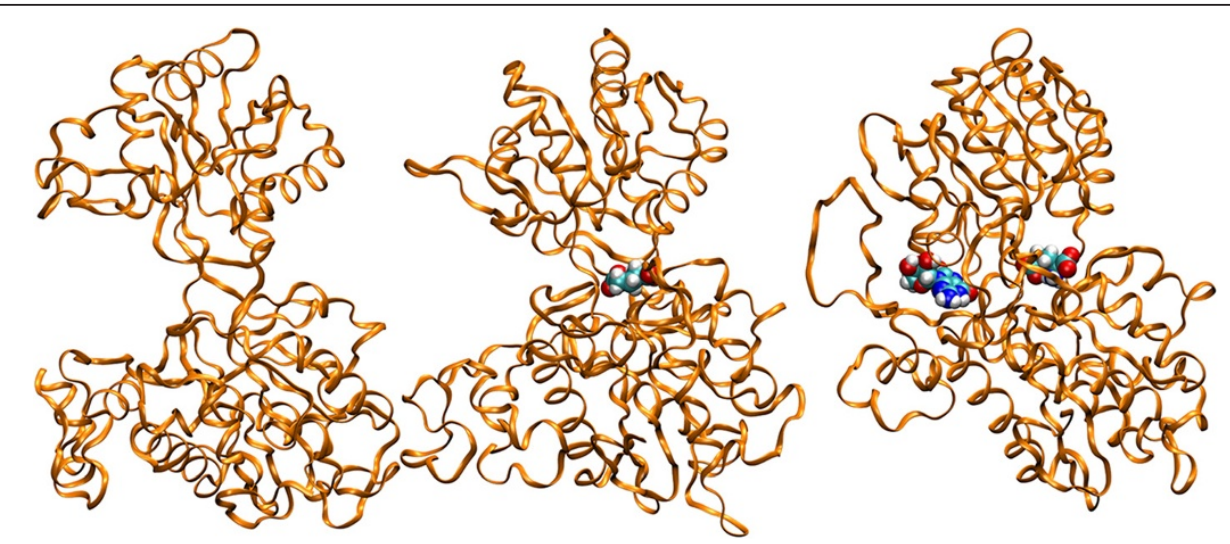

Figure 3 Molecular mechanism of the umami receptor. Ribbon-band representation of the Venus-fly trap motif on the T1R1/T1R3 umami receptor in three situations. From left: no bound ligands; binding of glutamate; binding of both glutamate and GMP. The images are snapshots obtained form molecular dynamics simulations on a receptor motif with 7,105 atoms in a box with 25,542 water molecules (not shown). 
this exciting mechanism arises as a consequence of an allosteric molecular action at the receptor.

The findings of our gastrophysical research program on seaweeds and umami holds promise for the design of novel compounds to controlling the umami flavoring of foodstuffs. Furthermore, our findings regarding the mechanism of the umami taste at the receptor level may not only lead to inspiration for new delicious dishes but may indicate how best to use umami as a means to regulate food intake and to improve nutrition and health [25].

\section{Competing interests}

The authors declare that they have no competing interests.

\section{Authors' contributions}

OGM designed the study and wrote the paper. LD performed the analyses of glutamate contents in dashi. LAB made the fluorescence microscopy images of the red seaweed dulse. HK performed the Molecular Dynamics simulations of the umami receptor. All authors read and approved the final manuscript.

\section{Acknowledgements}

MEMPHYS is supported as a center of excellence by the Danish National Research. HK is funded by a Lundbeck Junior Group Leader Fellowship. The computations were performed at the Danish Center for Scientific Computing at the University of Southern Denmark.

\section{Author details}

${ }^{1}$ MEMPHYS, Center for Biomembrane Physics, Department of Physics, Chemistry, and Pharmacy, University of Southern Denmark, Campusvej 55, Odense M DK-5230, Denmark. ${ }^{2}$ MEMPHYS, Center for Biomembrane Physics, Department of Biochemistry and Molecular Biology, University of Southern Denmark, Campusvej 55, Odense M DK-5230, Denmark.

Received: 8 November 2012 Accepted: 8 November 2012

Published: 9 January 2013

\section{References}

1. Myhrvold N, Young C, Bilet M: Modernist Cuisine: The Art and Science of Cooking, Volume 6. Bellevue, WA: The Cooking Lab; 2011.

2. Barham P, Skibsted LH, Bredie WLP, Bom Frøst M, Møller P, Risbo J, Snitkjær

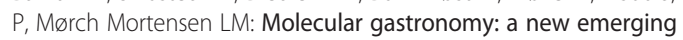
scientific discipline. Chem Rev 2010, 110:2313-2365.

3. Barham P: The Science of Cooking. Heidelberg: Springer; 2001.

4. McGee H: On Food and Cooking: The Science and Lore of the Kitchen. Revised edition. New York: Scribner; 2004.

5. Vilgis T: Das Molekül-Menü. Stuttgart: Hirzel; 2011.

6. Mouritsen OG: Sushi: Food for the Eye, the Body, \& the Soul. New York: Springer; 2009.

7. Mouritsen OG: Seaweeds. Edible, Available and Sustainable. Chicago: University of Chicago Press; 2013.

8. Blumenthal H, Barbot P, Matsushisa N, Mikuni K: Dashi and Umami: The Heart of Japanese Cuisine. London: Eat-Japan, Cross Media Ltd; 2009.

9. Mouritsen OG, Styrbæk K: Umami: Gourmetaben og den Femte Smag. [Umami: The Gourmetape and the Fifth Taste.]. Copenhagen: Nyt Nordisk Forlag Arnold Busck; 2011.

10. Ikeda I: New seasonings. Chem Senses 2002, 27:847-849.

11. Chaudhari N, Landin AM, Roper SD: A novel metabotropic glutamate receptor functions as a taste receptor. Nature Neurosci 2000, 3:113-119.

12. Kunishima N, Shimada Y, Tsuji Y, Sato T, Yamamoto M, Kumasaka T, Nakanishi S, Jingami H, Morikawa K: Structural basis of glutamate recognition by a dimeric metabotropic glutamate receptor. Nature 2000 407:971-977.

13. Nelson G, Chandrashekar J, Moon MA, Feng L, Zhao G, Ryba NJ, Zucker CS: An amino-acid taste receptor. Nature 2002, 416:199-202.

14. Li X, Staszewski L, Xu H, Durick K, Zoller M, Adler E: Human receptors for sweet and umami taste. Proc Natl Acad Sci U S A 2002, 99:4692-4696.

15. Humphries C: Cooking: delicious science. Nature 2012, 486:S10-S11.
16. Mouritsen OG: The emerging science of gastrophysics and its application to the algal cuisine. Flavour 2012, 1:6

17. Mouritsen OG, Williams L, Bjerregaard R, Duelund L: Seaweeds for umami flavour in the new Nordic cuisine. Flavour 2012, 1:4

18. Ninomiya K: Umami: a universal taste. Food Rev Int 2002, 18:23-38.

19. Ninomiya K: Natural occurrence. Food Rev Int 1998, 14:177-211.

20. Maga JA: Flavor potentiators. Crit Rev Food Sci Nutr 1993, 18:231-312.

21. Chandrashekar J, Hoon MA, Ryba NJ, Zucker CA: The receptors and cells for mammalian taste. Nature 2006, 444:288-294.

22. Mouritsen $\mathrm{OG}$, Khandelia $\mathrm{H}$ : Molecular mechanism of the allosteric enhancement of the umami taste sensation. FEBS J 2012, 279:3112-3120.

23. Zhang FB, Klebansky B, Fine RM, Xu H, Pronin A, Liu H, Tachdjian C, Li X: Molecular mechanism for the umami taste synergism. Proc Natl Acad Sci U S A 2008, 105:20930-20934.

24. Kuninaka A: Studies on taste of ribonucleic acid derivatives. J Agric Chem Soc Jpn 1960, 34:487-492.

25. Mouritsen OG: Umami flavour as a means to regulate food intake and to improve nutrition and health. Nutr Health 2012, 21:56-75.

doi:10.1186/2044-7248-2-9

Cite this article as: Mouritsen et al:: The name of deliciousness and the gastrophysics behind it. Flavour 2013 2:9.

\section{Submit your next manuscript to BioMed Central and take full advantage of:}

- Convenient online submission

- Thorough peer review

- No space constraints or color figure charges

- Immediate publication on acceptance

- Inclusion in PubMed, CAS, Scopus and Google Scholar

- Research which is freely available for redistribution 